Petra Lucht

Zur Herstellung epistemischer Autorität 
Soziologische Studien

Band 30 


\title{
Zur Herstellung epistemischer Autorität
}

\section{Eine wissenssoziologische Studie über die Physik an einer Elite-Universität in den USA}

\author{
Petra Lucht
}

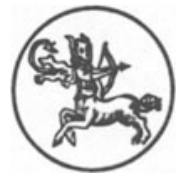

Centaurus Verlag \& Media UG 2004 


\section{Die Autorin:}

Petra Lucht, geb. 1967, ist Diplom-Physikerin und promovierte Soziologin. Zu ihren Arbeitsschwerpunkten gehören die Wissenschaftsforschung, die Frauen- und Geschlechterforschung über die Natur- und Technikwissenschaften und die qualitativen Methoden der Sozialforschung.

\section{Die Deutsche Bibliothek - CIP-Einheitsaufnahme}

Lucht, Petra:

Zur Herstellung epistemischer Autorität : Eine wissenssoziologische Studie über die Physik an einer Elite-Universität in den USA / Petra Lucht. Herbolzheim : Centaurus-Verl., 2004

(Soziologische Studien ; Bd. 30)

Zugl.: Hamburg, Univ., Diss., 2003

ISBN 978-3-8255-0514-1 ISBN 978-3-86226-505-3 (eBook)

DOI 10.1007/978-3-86226-505-3

\section{ISSN 0937-664X}

Alle Rechte, insbesondere das Recht der Vervielfältigung und Verbreitung sowie der Übersetzung, vorbehalten. Kein Teil des Werkes darf in irgendeiner Form (durch Fotokopie, Mikrofilm oder ein anderes Verfahren) ohne schriftliche Genehmigung des Verlages reproduziert oder unter Verwendung elektronischer Systeme verarbeitet, vervielfältigt oder verbreitet werden.

(C) CENTAURUS Verlags-GmbH. \& Co. KG, Herbolzheim 2004

Satz: Vorlage der Autorin

Umschlaggestaltung: Jörg Meyer, Kiel 


\section{Inhalt}

Vorwort

xiii

Danksagun $_{5}$

xix

Teil I Theoretische Einordnung und Forschungsstand

1 Theoretische Ausgangspunkte und Übersicht

1.1 Theoretische Ausgangspunkte und Fragestellung 3

1.1.1 Epistemische Autorität 3

1.1.2 Grenzziehungsarbeit zur Konstruktion epistemischer Autorität 5

1.1.3 Zur Verwendung des Begriffs 'Wissenschaftsverständnis der Physik' und die Fragestellung dieser Arbeit

1.1.4 'Grenzobjekte' und 'Grenzprojekte' als Konzepte zur

Beschreibung von Stabilität und Wandel von Wissensterritorien

1.2 Übersicht über die nachfolgenden Kapitel

2 Zum 'Wissenschaftsverständnis der Physik' in der Wissenschaftsforschung und der Frauen- und Geschlechterforschung

2.1 Von der Wissenssoziologie zur Wissenschaftsforschung

2.1.1 Der epistemologische Sonderstatus der Naturwissenschaften in Wissenssoziologie und Wissenschaftssoziologie

2.1.1.1 Das Abgrenzungsproblem in der Wissenssoziologie von Karl Mannheim

2.1.1.2 Abgrenzungskriterien in der Wissenschaftssoziologie 
2.1.2 Die soziale Konstruktion naturwissenschaftlicher Erkenntnis 27

2.1.2.1 Die 'Antipositivistische Wende' in der Wissenschaftstheorie 27

2.1.2.2 Soziologie des naturwissenschaftlichen Wissens 29

2.1.2.3 Naturwissenschaft als Praxis: Der Laborstudien-Ansatz 32

2.1.3 Reflexivitätskonzepte in der Wissenschaftsforschung 35

2.2 Frauen- und Geschlechterforschung über die Physik 38

2.2.1 Berufspolitische Dimensionen 39

2.2.1.1 Die Beteiligung von Frauen an der Physik in den Anfängen des Frauenstudiums

2.2.1.2 Der Anteil von Frauen an der Physik Mitte der 1990er Jahre in den USA

2.2.1.3 Das Wissenschaftsverständnis der Physik vor dem Hintergrund der berufspolitischen Dimensionen für Frauen

2.2.2 Das 'Soziokulturelle Geschlecht' in Konstruktionsprozessen physikalischen Wissens

2.2.2.1 Metaphernanalysen: 'Gender and Science' als 'Grenzfall' par excellence

2.2.2.2 Konstitution von geschlechtskonnotierten Kategorien in der physikalischen Theoriebildung

2.3 Fazit: Grenzverschiebungen und Kreuzungspunkte

3 Das Wissenschaftsverständnis von PhysikerInnen: Relevanz und Konzeption einer Interviewstudie mit Doktorandinnen und Doktoranden

3.1 Studien über das Wissenschaftsverständnis von PhysikerInnen in der Ausbildung

3.1.1 Das Wissenschaftsverständnis von PhysikerInnen in der Ausbildung: Ein blinder Fleck der Wissenschaftsforschung und seine Folgen

3.1.1.1 Ethnographische Untersuchungen über Forschungsinstitute der Physik: Ergebnisse über die Vermittlung von Vorstellungen von der Physik durch Studien- und Forschungsstrukturen 
3.1.1.2 Auswahlkritierien für die empirische Erhebung

3.1.2 Studien der Schul- und Hochschulforschung zu Vorstellungen von den Naturwissenschaften von SchülerInnen und Studierenden der Physik

3.1.2.1 Charakterisierung der exemplarisch betrachteten Studien

3.1.2.2 Vorstellungen von den Naturwissenschaften im Zusammenhang mit Zielen naturwissenschaftlichen Unterrichts

3.1.2.3 Anschlussfähige Ergebnisse

3.1.2.4 Dimensionen des Wissenschaftsverständnisses im Hinblick auf die Wissenschaftsforschung

3.1.2.5 Die Vermittlung von Vorstellungen von der Physik durch institutionalisierte Unterrichtsstrukturen in der High School

3.1.3 Zwischenfazit aufgrund des Forschungsstands

3.2 Methodologischer Begründungsrahmen und die Verfahren der empirischen Erhebung

3.2.1 Methodologischer Begründungsrahmen: Die SozialforscherIn als Babelfisch in der Postmoderne

3.2.1.1 Qualitative Sozialforschung als Prozess

3.2.1.2 Postmoderne Subjekte in der qualitativen, empirischen Sozialforschung

3.2.1.3 Partiale, situative Übersetzungen im Forschungsprozess 108

$\begin{array}{ll}\text { 3.2.2 Phasen des Forschungsprozesses } & 114\end{array}$

$\begin{array}{lll}\text { 3.2.2.1 Das Erhebungsdesign } & 114\end{array}$

$\begin{array}{ll}\text { 3.2.2.2 Auswertung der Interviews } & 129\end{array}$

3.2.3 Zwischenfazit zum methodischen Vorgehen 142

3.3 Fazit 
Teil II Ergebnisse der empirischen Erhebung

4 Institutionalisierte Grenzziehungen in Studium und Forschung

4.1 Grenzziehung zwischen Undergraduate- und Graduate-Studium: Der Übergang von einer formal zu einer informell strukturierten Ausbildungsphase

4.1.1 Zur Historie des Promotionsstudiums in den USA

4.1.2 "It's been a real education for me, to make that transition." Grenzziehungen zwischen Undergraduate- und GraduateStudium aus der Perspektive der interviewten Doktorandinnen und Doktoranden

4.1.2.1 Die Differenz von Undergraduate- und Graduate-Studium als In-vivo-Kode

4.1.2.2 Zeitaufwand und -management: "Well, [life] is pretty busy, but it's pretty free."

4.1.2.3 Fähigkeiten

4.1.2.4 Anerkennung

4.1.2.5 Wissenschaftsverständnis: Von der 'Lehrbuchanalyse' zum 'Forschungsdesign'?

4.1.3 Der Übergang vom Undergraduate- zum Graduate-Studium: Grenzziehungen zwischen einer formalisierten und einer informell strukturierten Ausbildungsphase

4.1.3.1 Zur individuellen Gestaltung von Forschungsprozessen im Graduate-Studium

4.1.3.2 Der Ausschluss der „Anderen“ aufgrund informeller Zugangsund Qualifikationskriterien am Beispiel der Beteiligung von Frauen

4.1.4 Zwischenfazit 
4.2 Zur Differenzierung von theoretischer und experimenteller Physik

4.2.1 Grenzziehungen zwischen theoretischer und experimenteller Physik aus der Perspektive der interviewten Doktorandinnen und Doktoranden

4.2.1.1 Die Differenzierung von theoretischer und experimenteller Physik als In-vivo-Kode

4.2.1.2 Das Selbstverständnis der Interviewten

4.2.1.3 Wissenschaftsverständnis

4.2.2 Institutionalisierung von theoretischer und experimenteller Physik

4.2.2.1 Die Vergabe von Studienplätzen an der Waterside University an Promovierende der Physik

4.2.3 Zwischenfazit: Institutionalisierter Positivismus

4.3 Fazit

$5 \quad$ Physik als Zunft

5.1 Die Konzepte der 'Zunft' und der 'Lehrzeit'

5.2 Die Regeln der Zunft an der Waterside University

5.2.1 Das 'Empfehlungssystem'

5.2.2 Das Lernen, Lehren und Forschen im Beziehungsgefüge der Zunft

5.2.2.1 Promovierende und ihre BetreuerInnen

5.2.2.2 Die Zusammenarbeit mit anderen ForscherInnen 209

5.2.2.3 Zusammenarbeit mit anderen DoktorandInnen 211

$\begin{array}{ll}\text { 5.2.2.4 'Totale Teamarbeit' } & 212\end{array}$

5.2.3 Das 'Persönliche' wiegt schwerer als das 'Fachliche' 215

5.2.3.1 Die Persönlichkeit des Betreuers oder der Betreuerin 216

5.2.4 Finanzielle Ressourcen für Promovierende 221

5.2.4.1 Beteiligung an der Lehre der Waterside University 223

5.2.4.2 Forschungsgelder aus der Arbeitsgruppe 225 
5.2.5 Promovieren als Prozess: Die Phasen der Lehrzeit

5.2.5.1 Vor dem Einstieg in eine Arbeitsgruppe: Erste Forschungserfahrungen

5.2.5.2 Anfangsphase der Lehrzeit

5.2.5.3 Die mittlere Phase: Konsolidierung

5.2.5.4 Die Endphase des Promovierens als Aufnahme in die Zunft

5.2.5.5 Störungen des Prozesses der Lehrzeit aufgrund eines Wechsels der Betreuung

5.2.5.6 Zwischenfazit zur Lehrzeit als Prozess

5.3 Das Gesellenstück: Die Dissertation als 'Grenzobjekt'

5.3.1 Die Dissertation als Qualifizierungsarbeit

5.3.2 Die Dissertation als Beitrag zur physikalischen Forschung

5.3.2.1 Fragestellungen in der Physik

5.3.2.2 Interdisziplinäre Fragestellungen

5.3.2.3 Nicht physikalisch arbeiten, um einen Doktortitel in der Physik zu erwerben: ein Paradox?

5.3.3 Die Geschlechtskonnotation des Doktortitels 256

5.3.4 Wem gehört das physikalische Wissen? 262

$\begin{array}{lll}5.4 & \text { Fazit } & 266\end{array}$

6 Von der Kunst, Geschichten zu erzählen 271

6.1 Historisierende Erzählungen: "He has particles named after him."

6.1.1 Historisierung eines Forschungsgebiets oder -projekts anhand der Erfolge berühmter PhysikerInnen

6.1.2 Die Historie der Physik als Erbschaft: Identifikation und Abgrenzung

6.1.2.1 David: "I do feel like I'm in the direct line of things that happened from Einstein on."

6.1.2.2 Maria: "I never really expected [...] that I would become some great physicist." 
6.2.1 Die Ausbildungsperspektive: Die Bedeutung populärwissenschaftlicher Darstellungen für die eigene Berufsplanung

6.2.2 Die Forschungsperspektive: Presseberichte über aktuelle Ergebnisse

6.3 "To travel faster than light": Imaginationen, Sciencefiction und die Grenzen der Physik

6.3.1 Frühes Interesse an Sciencefiction-Geschichten

6.3.2 Sciencefiction als Mittel zur Kommunikation von Forschungsinhalten

6.3.3 Vorstellungen von zukünftigen Forschungen der Physik 306

6.3.4 Zwischenfazit: Sciencefiction als Grenzprojekt

6.4 Fazit: Spekulationen zur Wirkmächtigkeit tradierter Geschichten

$7 \quad$ Zur »Herstellung epistemischer Autorität«

7.1 Zusammenfassung der Ergebnisse

7.2 Synopse: Zur »Herstellung epistemischer Autorität»

7.2.1 Daten und Theorien über die Natur sind ,institutionsgeladen“.

7.2.2 Stabilität und Wandel von Klassifikationen und Wissensterritorien

7.2.3 Das Klassizifieren erlernen

7.2.4 Reflexivität als Voraussetzung für die »Herstellung epistemischer Autorität« 
$8 \quad$ Literatur

9 Anhang $\quad 347$

9.1 Abkürzungen und Hervorhebungen 347

9.1.1 Abkürzungen und Hervorhebungen im Text 347

9.1.2 Abkürzungen und Hervorhebungen in Interview-Transkripten 347

9.2 Interviewstudie 349

9.2.1 Ankündigung der Studie (Aushang) 349

9.2.2 Einverständniserklärung für die Teilnahme an der

9.2.3 Veröffentlichung von Zitaten 350

$\begin{array}{lll}\text { 9.2.4 Interviewleitfäden } & 350\end{array}$

9.2.4.1 Interviewleitfaden (englische Version) $\quad 350$

9.2.4.2 Interviewleitfaden (deutsche Version) 353 


\section{Vorwort}

Die hier dargelegte Untersuchung habe ich in der Wissenschaftsforschung und in der Frauen- und Geschlechterforschung über die Physik verortet. In diesen Bereichen sind Ansätze entwickelt worden, um das ,Soziale' - das jedoch sehr unterschiedlich gefasst wird - als integralen Bestandteil naturwissenschaftlicher Erkenntnisproduktion zu untersuchen. Beide Forschungsfelder habe ich insbesondere für den bundesdeutschen und den anglo-amerikanischen Kontext exemplarisch anhand unterschiedlicher Ausrichtungen bzw. ihrer prominenten VertreterInnen aufgearbeitet.

Bis auf wenige Ausnahmen mangelt es diesen Studien zumeist daran, Dialoge mit den Beteiligten an den untersuchten Disziplinen, also mit Naturwissenschaftlerinnen und Naturwissenschaftlern zu führen oder diese ausführlich zu dokumentieren. Zudem stellt die Untersuchung der Ausbildung von NaturwissenschaftlerInnen eine eklatante Forschungslücke in der Wissenschaftsforschung dar, obwohl es auch und gerade die Ausbildung ist, anhand derer sich Bedingungen für die soziale Produktion und Reproduktion von Wissen einer Wissenschaft analysieren lassen. Mit dieser Studie leiste ich einen Beitrag zu diesen beiden Forschungsdesideraten. Um NaturwissenschaftlerInnen zu Wort kommen zu lassen und zugleich die Ausbildung in den Naturwissenschaften analysieren zu können, habe ich den Dialog mit NaturwissenschaftlerInnen auf methodischkonzeptioneller Ebene in Form einer qualitativen Interviewstudie mit PhysikDoktorandinnen und -Doktoranden einer Elite-Universität in den USA, der 'Waterside University', gesucht.

Auch wenn Teilnehmende an einer qualitativen Interviewstudie ausführlich zu Wort kommen, ist der dialogische Charakter einer solchen Untersuchung begrenzt, da das Forschungsdesign so angelegt ist, dass ihm strukturelle Asymmetrien und Machtkonstellationen zwischen Teilnehmenden und Forschenden zugunsten letzterer inne wohnen. Das Deutungsmonopol über die Äußerungen der Interviewten liegt bei der SozialforscherIn als AnalysandIn und AutorIn von Publikationen. Gegengewichte zu diesem Deutungsmonopol habe ich insofern zu setzen gesucht, als ich ausführliche Zitate der DoktorandInnen, die einen reflexiven Charakter in Bezug auf die Wissensproduktion und -reproduktion in der Physik aufweisen, im Rahmen der Vorstellung der Ergebnisse im zweiten Teil

Um die Anonymität der Interviewten zu wahren, wurde der Name der untersuchten Universität durch eine frei erfundene Bezeichnung ersetzt. 
meiner Untersuchung angeführt habe. Dies soll zum einen die wichtige Rolle der Äußerungen der Interviewten für diese Arbeit hervorheben und zum anderen den Leserinnen und Lesern ermöglichen, meine Analysen nachzuvollziehen und kritisieren zu können.

Wie sind die in dieser Studie angeführten Äußerungen der Interviewten, meine weitergehenden Analysen und die hieran anschließenden Schlussfolgerungen $\mathrm{zu}$ verstehen? Um diese Frage zu beantworten, habe ich es als notwendig angesehen, vor der Darlegung der empirischen Erhebung mein methodologisches Selbstverständnis zu klären. Im Anschluss an das Konzept des 'Situierten Wissens' der Wissenschaftsforscherin Donna Haraway ([1988] 1991), das m. W. bislang kaum hinsichtlich seiner Ausdeutungen für die Methodenentwicklung in den Sozialwissenschaften aufgegriffen worden ist, verstehe ich die Bearbeitung einzelner Arbeitsschritte eines qualitativen Forschungsprojekts als auch die in diesem Falle erhobenen Äußerungen von TeilnehmerInnen an einer Interviewstudie als sich permanent vollziehende, situative und partiale Übersetzungen. Diese Übersetzungen sind nicht frei von meinen Wertungen, auch geben sie nicht in vollständiger Weise die Perspektiven der Interviewten auf ihre Alltagswelt wieder. Vielmehr verstehe ich meine Arbeit so, dass ich die Äußerungen der von mir interviewten Doktorandinnen und Doktoranden der Physik in mehreren Arbeitsschritten übersetzt und damit eine weitere wissenssoziologische - situative und partiale - Erzählung über die Physik entworfen habe.

Für meine Arbeit sind gegenüber einer Wissenssoziologie Mannheim'scher Prägung zumindest drei zentrale Verschiebungen zu berücksichtigen: Während Mannheim die Naturwissenschaften und die Mathematik von einer Seinsverbundeheit des Wissens ausnimmt, fasse ich auch die Wissensproduktion in diesen Disziplinen als „,seinsverbunden“, $d$. h. als ein sozial konstruiertes auf und stelle mich damit in die Tradition von Ansätzen der Wissenschaftsforschung, die nach der sogenannten 'Antipositivistischen Wende' entwickelt worden sind (vgl. auch Abschnitt 2.1). Die zweite Verschiebung gegenüber dem Mannheim'schen Konzept der Wissenssoziologie besteht darin, dass ich nicht von einer eindeutig festgelegten Standortverbundenheit des Wissens ausgehe, sondern naturwissenschaftliches wie auch sozialwissenschaftliches Wissen im Anschluss an Haraway als partial situiertes auffasse. Als dritte Verschiebung gegenüber der Mannheim'schen Position ist es nicht mein Anliegen, in dieser Arbeit eine Seinsverbundenheit des Wissens per se nachzuweisen, sondern meine Fragestellung ist darauf ausgerichtet unterschiedliche Auffassungen über die soziokulturelle Herstellung naturwissenschaftlichen Wissens anhand von Äußerungen von Naturwissenschaftlerinnen und Naturwissenschaftlern herauszuarbeiten. Der Zusam- 
menhang zwischen sozialer Verortung der Teilnehmerinnen und Teilnehmer an meiner Studie und ihren Auffassungen über die Wissensproduktion in der Physik wird $\mathrm{m}$. E. durch ihre in den Interviews immer wieder aufscheinende Reflexivität gebrochen, so dass von einer sozialen Verortung im Wissenschaftsbetrieb nicht nahtlos auf bestimmte Auffassungen über die Wissensproduktion rückgeschlossen werden kann. Ich komme hierauf noch zurück.

Um deutlich zwischen der Rekonstruktion der Perspektiven der Interviewten und meinen Deutungen zu unterscheiden, habe ich weiterhin, den Prämissen der 'Grounded Theory' folgend, zwischen zwei Formen der Kategorienbildung unterschieden: Anhand des Interviewmaterials habe ich zunächst die in Bezug auf meine Fragestellung relevanten In-vivo-Kodes festgelegt, d. h. Kategorien, die von den Interviewten verwendet wurden, um ihre Perspektiven auf die Physik zu darzustellen. Um Missverständnissen zu begegnen, möchte ich hier betonen, dass ich also für diesen ersten Analyseschritt nicht davon ausgehe, dass diese In-vivoKodes aus dem Datenmaterial ohne weitere Vorannahmen emergieren könnten. Vielmehr setzt bereits dieser erste Analyseschritt eine Bewertung des Materials von meiner Seite voraus. In einem zweiten Schritt habe ich dann die herausgearbeiteten In-vivo-Kodes nochmals unter veränderten Perspektiven durch soziologische (Um-)Deutungen erweitert bzw. kontrastiert.

Die Ergebnisse meiner Untersuchung weisen darauf hin, dass die interviewten Promovierenden der Physik v. a. diejenigen sozialen Konstruktionsprozesse der Wissensproduktion in der Physik thematisieren, die auf der Mesoebene von institutionalisierten Ausbildungs- und Forschungsstrukturen der untersuchten Universität aufzufinden sind. Aus- und Einschlüsse von Personen und Wissensformen vollziehen sich nicht nur an den ,äußeren Grenzen“ dieser Institution, d. h. beispielsweise im Rahmen von Bewerbungen um einen Studienplatz oder von Zwischen- und Abschlussprüfungen. Vielmehr werden unterschiedliche Ausbildungsphasen und Forschungsgebiete in hierarchisierender Weise zueinander angeordnet. Diese Strukturen werden von den interviewten DoktorandInnen nachvollzogen und reproduziert, da sie in eben diesen Strukturen auch selbst verortet sind. So werden beispielsweise das Promotions- gegenüber dem CollegeStudium und die theoretische gegenüber der experimentellen Physik jeweils höher bewertet. Dass sich dies auf Ein- und Ausschlüsse von marginalisierten sozialen Gruppen in der Physik auswirkt, kann anhand der Beteiligung von Frauen an diesen Ausbildungsphasen bzw. Forschungsgebieten aufgezeigt werden, denn der Anteil von Doktorandinnen ist in den höher bewerteten Bereichen geringer als in der niedriger bewerteten. Diese Zuordnungsprinzipien waren in 
meiner Studie relativ gut zu erfassen, da diese Ausbildungsphasen bzw. Forschungsgebiete zumeist auch formal institutionalisiert sind und an der 'Waterside University' Daten über entsprechende Anteile von unterschiedlichen sozialen Gruppen an einzelnen Forschungsgebieten erfasst werden.

Weniger offensichtlich ist, welche Ein- und Ausschlussmechanismen aufgrund informeller Sozialstrukturen an einer untersuchten Institution wirksam werden. Für die Deutung dieser Mechanismen habe ich in Erweiterung einer Erzählung von einem der interviewten Doktoranden das Analysekonzept der 'Physik als Zunft' eingeführt. Diese flexible und zugleich ordnende Sozialstruktur erlaubt es den Beteiligten an der Disziplin, auf vielfältigen informellen Wegen zu verhandeln, welche Wissensformen als zur Physik zugehörig angesehen werden und welche Personen in der Physik promovieren können. Für einen gleichberechtigten Zugang unterschiedlicher sozialer Gruppen bringt jedoch diese informelle Sozialstruktur mehr Nachteile als Vorteile mit sich, da tradierte Vorstellungen über die Disziplin und über die an ihr Beteiligten $\mathrm{m}$. E. tendenziell reproduziert werden. So äußern die Interviewten bezogen auf die Beteiligung von Frauen an der Physik während der Phase des Promovierens, dass das Berufsbild von der Physik als einer Männerdomäne nach wie vor prägend ist. Der Doktortitel ist damit bis heute trotz der zunehmenden Beteiligung von Frauen an den Wissenschaften männlich konnotiert, da er, so einer der von mir interviewten Doktoranden, „sexistische Wurzeln“ habe und „Männern des Wissens“ mehr „Respekt“ verleihe.

Auf diskursiver Ebene werden hierarchische Anordnungen durch 'Tradierte Geschichten', d. h. mündlich oder schriftlich weitergegebene Erzählungen über die Physik, reproduziert und bestätigt. Diese Geschichten aus den Genres der historisierenden und der populärwissenschaftlichen Erzählungen sowie den Sciencefiction erfüllen unterschiedliche Funktionen für die interviewten DoktorandInnen. Sie „begleiten“ die Promovierenden z. T. quasi von Kindheit an auf ihrem Karriereweg in die Physik. So äußern TeilnehmerInnen an meiner Untersuchung, dass diese Erzählungen sie dazu motiviert hätten, ein Physikstudium zu beginnen oder dass sie bestimmte Vorstellungen aus Sciencfiction-Filmen verwendeten, um physikalische Forschungsinhalte mithilfe von Metaphern zu vermitteln. Auch prägen 'Tradierte Geschichten' teilweise die Vorstellungen der Promovierenden über zukünftige Forschungsprojekte. 'Tradierten Geschichten' ist häufig gemeinsam, dass darin berühmte, zumeist männliche PhysikerInnen eine zentrale Rolle als Experten des physikalischen Wissens spielen. Die Reproduktion tradierter Sozialstrukturen spiegelt sich pointiert in einer Erzählung eines Doktoranden wieder, der sich selbst als einen „Neffen Einsteins“ empfindet und sich damit in 
dessen soziokultureller Nachfolge sieht - sein Erbe antretend. Für die Frage nach der Beteiligung von Frauen gewendet bedeutet dieses Ergebnis meiner Untersuchung, dass es um Veränderungen von Vorstellungen soziokultureller (Erb-) Nachfolge bei denjenigen in der Disziplin der Physik gehen muss, die über kommende Nachfolgerinnen und Nachfolger in der Disziplin entscheiden.

Die Ergebnisse meiner Untersuchung habe ich in Kapitel 7 abschließend und in Anlehnung an die 'Grounded Theory' in Form einer analytischen Erzählung über die anhand des erhobenen Materials herausgestellten Kategorien synoptisch zusammengeführt. Dort formuliere ich thesenartig einige Charakteristika und Voraussetzungen der Kernkategorie dieser Arbeit, nämlich der »Herstellung epistemischer Autorität«, d. h. einer auf Wissen beruhenden Autorität: Unter Bezugnahme auf die herausgearbeiteten Sozialstrukturen gehe ich davon aus, dass Daten und Theorien in den Naturwissenschaften ,institutionsgeladen“ sind, da soziale Konstruktionsprozesse physikalischen Wissens auf der Ebene von Institutionen stattfinden. Weiterhin führe ich im Verlauf der Arbeit die analytischen Konzepte von 'Grenzobjekten' und 'Grenzprojekten' als polarisiertes Begriffspaar ein, um Stabilität und Wandel von auffindbaren institutionalisierten Klassifikationen und Wissensterritorien zu plausibilisieren. Beide Begriffe konkretisiere ich, indem ich beispielsweise Dissertationen tendenziell als 'Grenzobjekte' ansehe, die zu einer Stabilisierung von bestehenden Klassifikationen und Wissensterritorien beitragen, während ich demgegenüber Sciencefiction-Geschichten als 'Grenzprojekte' einordne, die die Grenzen des bestehenden Wissenskanons der Physik infrage stellen, indem sie Imaginationen für Veränderungen bestehender Klassifikationen und Wissensterritorien der Physik bereit stellen. Ein drittes Charakteristikum für die Herstellung epistemischer Autorität ist, dass das „Klassifizieren" erlernt wird. In meiner Untersuchung zeigt sich dies daran, dass Promovierende durch ihre eigene Verortung innerhalb der disziplinspezifischen Klassifikationen und Wissensterritorien lernen, diese nachzuvollziehen und zu reproduzieren. Epistemische Autorität ist damit nicht notwendigerweise an bestimmte Personen gebunden, sondern auch an institutionalisierte Strukturen und stellt somit eine Form von abstrakter Autorität dar. Der Prozess der Herstellung epistemischer Autorität vollzieht sich für die interviewten DoktorandInnen aber zugleich auch auf individueller Ebene, indem sie einen Doktortitel anstreben, der ihnen eine bestimmte Ausprägung epistemischer Autorität verleihen wird. Meine vierte These ist, dass Reflexivität als Voraussetzung für die Herstellung epistemischer Autorität anzusehen ist. Den Begriff der 'Reflexivität' fasse ich in dieser Untersuchung folgendermaßen: In dem Datenmaterial meiner Studie sind viele 
Äußerungen der Interviewten aufzufinden, mit denen sie sich von vorherigen oder anschließenden Äußerungen distanzieren. Diese 'Metastatements' über ihre Erzählungen geben Hinweise darauf, dass die Interviewten die von ihnen vorgefundenen Kategorien, so auch die Klassifizierungen und Wissensterritorien in der Physik, zugleich distanzierend und damit reflektierend bewerten. Ich schließe mit meinen Analysen auch an diese Distanzierungen der Interviewten an. Dieses Verständnis des Begriffs 'Reflexivität' weicht insofern von der Verwendung dieses Begriffs in der Wissenschaftsforschung ab, als Ansätze letzterer zumeist davon ausgehen, dass die Natur- und Technikwissenschaft nicht (selbst-)reflexiv seien. Zumeist meint diese Zuschreibung, dass in den Natur- und Technikwissenschaften die eigene soziale Verortung nicht reflektiert werde. Damit wird jedoch ein Unterscheidungsmerkmal zwischen den zwei „Zwei Wissenschaftskulturen“ etabliert, das m. E. nicht als solches zu halten ist, wenn der Begriff 'Reflexivität' wie o. a. in erweiterter Form gefasst wird. Ausgehend von einer so verstandenen 'Reflexivität' sehe ich für die sozialwissenschaftlich orientierte Wissenschaftsforschung weiterführende Perspektiven darin, den Dialog mit Naturwissenschaftlerinnen und Naturwissenschaftlern zu suchen, ohne eigene Prämissen für die Untersuchung naturwissenschaftlicher Erkenntnisproduktion preis zu geben. In diesem Sinne plädiere ich mit meiner Untersuchung für einen fortgesetzten Dialog über die Grenzen der 'Two Cultures' hinweg. 


\section{Danksagung}

Diese Untersuchung wurde von zahlreichen Diskussionskontexten geprägt. Allen Personen, die mir mit offener Kritik, Anregungen, Enthusiasmus und Unterstützung begegnet sind, danke ich für ihre Zeit und ihre Auseinandersetzung mit diesem Projekt. Ihre Beiträge sind in vielfältiger Weise in den Forschungsprozess eingegangen.

Mein besonderer Dank gilt den ehemaligen Doktorandinnen und Doktoranden der Physik an der 'Waterside University' in den USA, die an der qualitativen Interviewstudie für diese Untersuchung teilgenommen haben. Ihre Erzählungen über die Physik haben mich fasziniert und beeindruckt - ich hoffe, mit meiner Darstellung ihrem Vertrauen gerecht geworden zu sein.

Prof. Dr. Ingrid N. Sommerkorn-Abrahams hat meinen Arbeitsprozess persönlich und fachlich in einer Weise begleitet, die es mir erlaubt hat, meine Vorstellungen und Ideen für dieses Projekt konsequent verfolgen und umsetzen zu können. Die Ausführung dieser Arbeit ist in ihren Kolloquien und in Veranstaltungen des Netzwerks LINT (Lehrende in Informatik, Naturwissenschaften und Technik) mehrfach diskutiert worden. Ihre Zusicherung, die Durchführung dieser Untersuchung am Institut für Soziologie der Universität Hamburg zu unterstützen, ist eine unerlässliche und motivierende Basis für mich gewesen. An diesem Institut ist das Projekt zudem unterstützt worden von Prof. Dr. Katharina Liebsch, die als ehemalige wissenschaftliche Assistentin weiterführende Kritik geübt hat, von Prof. Dr. Marianne Pieper, deren „Forschungswerkstatt Soziale Konstruktion von Körpern, Geschlechtern und Identitäten“ ein wichtiger Diskussionskontext in der Endphase des Projekts gewesen ist, von Prof. Dr. Max Miller, der sich sich mit den Ergebnissen und Thesen dieser Arbeit offen und kritisch auseinandergesetzt hat und von Prof. Dr. Marie-Elisabeth Hilger, die mich als Mentorin zu entscheidenden Zeitpunkten der Untersuchung in ausgezeichneter Weise begleitet hat.

Motiviert worden ist die hier vorliegende soziologische Untersuchung durch meinen biographischen Hintergrund als Diplom-Physikerin, als welche ich mich seit meiner Studienzeit permanent mit der Frage konfrontiert sah, warum so wenige Frauen an der Wissensproduktion in der Physik beteiligt sind. Ende der

2

Vgl. Fußnote 1. 
1980er bis Anfang der 1990er Jahre habe ich mich in einer Frauen-AG in der Physik an der Universität Kiel, durch die Mitarbeit an einem Buchprojekt mit feministischer Zielsetzung der Diplom-Physikerin Rosemarie Rübsamen in Hamburg sowie auf den „Bundesweiten Kongressen von Frauen in Naturwissenschaft und Technik" mit unterschiedlichen Ansätzen der Gender Studies über die Naturwissenschaften auseinandergesetzt, die sich damals noch weitgehend in der Entwicklung befanden. In Seminaren von Dr. Ursula Pasero habe ich meine Begeisterung für die Soziologie entdeckt und Ansätze der Frauen- und Geschlechterforschung über die Physik in offiziell anerkannten Lehrveranstaltungen an der Universität bearbeiten können. Meine frühen Entwürfe für ein Forschungsprojekt zur Untersuchung der Ursachen der geringen Beteiligung von Frauen an der Disziplin der Physik fanden an der Universität Kiel Unterstützung durch Prof. Dr. Gerd Wibberenz, Dr. Lore Hoffmann, Prof. Dr. Brigitte Lohff und Prof. Dr. Walter Westphal.

Mit der fortgesetzten Entwicklung dieser Arbeit wurde Dr. Heike Wiesner an der Universität Bremen zu einer Diskussionspartnerin, die meine Ideen und Texte kontinuierlich kommentiert und in kompetenter Weise konstruktiv kritisiert hat. Ihre freimütige Motivation hat mich bis zum Ende dieses Projekts begleitet. Anfang der 1990er Jahre habe ich den überregionalen „Arbeitskreis Feministische Naturwissenschaftsforschung und -kritik" (afn) mit begründet, der für mich bis heute ein Forum für wissenschaftlichen Austausch geblieben ist. Für Kritik und Anregungen zu meinen Texten aus diesem Kreis danke ich Corinna Bath, Prof. Dr. Smilla Ebeling, Dr. Helene Götschel, Dr. Dorit Heinsohn, Julia Lademann, Bärbel Mauß, Dr. Kerstin Palm, Barbara Petersen, Agnes Sandner, Ivana Weber und Dr. Heike Wiesner.

Ein großer Teil der konzeptionellen Ideen für diese Untersuchung geht auf meinen Aufenthalt 1994 - 1996 als Gastwissenschaftlerin an dem Program in Women's Studies des Massachusetts Institute of Technology (M.I.T.) zurück, den mir Prof. Evelyn Fox Keller ermöglicht hat. Diese Zeit hat zur Vertiefung meiner Kenntnisse der Frauen- und Geschlechterforschung über die Naturwissenschaften sehr beigetragen; zudem habe ich unterschiedliche Ansätze der Wissenschaftsforschung durch das ebenfalls am M.I.T. verankerte Program in Science, Technology and Society kennen gelernt. Vertiefende Diskussionen über Ansätze dieser beiden wissenschaftlichen Ausrichtungen habe ich dort in der „Working Group on Gender and Science“ (WoGGS) geführt. Mit ihren Ideen und Anregungen zur Konzeption des Projekts haben damals Leslie Barber, Ph.D., 
Prof. Rebecca Herzig, Prof. Vera Kistiakowsky, Prof. Mary Parlee, Prof. Karen Rader, Prof. Sherry Turkle und Nadine Weidman, Ph.D., beigetragen.

Für meine Auseinandersetzungen mit qualitativen Methoden der Sozialforschung sind v. a. meine Arbeiten an der Harvard Graduate School of Education der Harvard University von hervorzuhebender Bedeutung gewesen. Prof. Annie Rogers hat 1996 die Initiative ergriffen, mich als Gastwissenschaftlerin an dieses Institut einzuladen; mein weiterer Aufenthalt ist von Prof. Eleanor Duckworth unterstützt worden. Ausführliche Diskussionen habe ich dort mit TeilnehmerInnen von Seminaren von Prof. Annie Rogers und Prof. Sharon Rallis geführt, insbesondere mit Seeta Pai und Prof. Bonnie Tai. Auch hatte ich die außergewöhnliche Gelegenheit, zwei Forscherinnen, Fiona Hughes-M ${ }^{\mathrm{c} D}$ Donnell, Ed.D., und Elizabeth Cavicchi, Ed.D., zu treffen, die wie ich qualitative Interviewstudien mit SchülerInnen bzw. -Studierenden der Physik durchgeführt hatten, so dass wir unsere Untersuchungen vergleichend diskutieren konnten. Darüber hinausgehend haben wir Unterrichtskonzepte mit antiautoritärem Anspruch und für ein spielendes Erlernen von Erklärungen für physikalische Phänomene und Zusammenhänge am Beispiel von „Licht und Schatten“ entworfen.

Gegen Ende des Projekts hat Dr. Ursula Mihçiyazgan, Universität Hamburg, meinen Arbeitsprozess mit freundschaftlichen und kritisch-konstruktiven Anregungen, Kommentaren und Hinweisen sehr unterstützt. Für wissenschaftlichen Austausch über Zusammenhänge von Physik, Sciencefiction und Festkörperphysik danke ich Prof. Dr. Metin Tolan. Die LeserInnen von Teilen der Endfassung des Manuskripts waren Martina Erlemann, Stefanie Gräfe, Dr. Henrike Hölzer, Marion Mayer, Dr. Ursula Mihçiyazgan, Dr. Kerstin Palm, Dagmar Rohde, Ivana Weber, Dr. Heike Wiesner und Michaela Will, denen ich für ihre konstruktiven und weiterführenden Hinweise danke. Meiner Kollegin Ivana Weber danke ich für ihre Kritik und ihre Anregungen sowie für ihre herzliche und unterstützende Haltung während der Endphase dieses Projekts. Ein besonderer Dank gilt auch Prof. Dr. Werner Härdtle. Jörg Meyer entwarf das pointierte Titelbild.

Mein Partner Wolfgang Lucht hat alle wunderbaren und kritischen Momente dieses Projekts mitgetragen. Seine Begleitung war unsagbar wertvoll.

Während der Bearbeitung dieses Forschungsvorhabens erhielt ich Stipendien von der Universität Bremen, dem „Deutschen Akademischen Austauschdienst“ (DAAD), dem „Evangelischen Studienwerk Villigst e.V.“ und der Universität Hamburg (Stipendium des „Hochschulsonderprogramms III“). 